\title{
Bulk Metal Forming of Additively Manufactured Elements
}

\author{
Thomas Papke ${ }^{1 *}$, Daniel Junker ${ }^{1}$, Michael Schmidt ${ }^{2}$, Tobias Kolb ${ }^{2}$, and Marion Merklein ${ }^{1}$ \\ ${ }^{1}$ Friedrich-Alexander-Universität Erlangen-Nürnberg, Institute of Manufacturing Technology, Egerlandstr. 13, 91058 Erlangen, Germany \\ ${ }^{2}$ Friedrich-Alexander-Universität Erlangen-Nürnberg, Institute of Photonic Technologies, Konrad-Zuse-Str. 3, 91052 Erlangen, Germany
}

\begin{abstract}
Current trends in the industry go towards individualised parts in high volume production. Facing the high amount of different parts, efficient processes with a high flexibility are necessary. Within this context, laser-based additive manufacturing of metal parts become more important. These processes enable the production of individual geometries using a variety of metal materials. However, main challenges of this technology are rough surfaces, limited geometric precision and varying material properties. Therefore machining after the additive manufacturing process is a common approach for improving surface quality and geometric accuracy. However, the machining process affects the grain structure at the edges of the part. Using forming instead leads to a deformation of the grains but does not create new interfaces. Furthermore, the material properties can be improved due to work hardening and fibre flow. Within this work, a bulk metal forming process of an additively manufactured element of stainless steel $316 \mathrm{~L}$ will be analysed. The aim of the investigation is a fundamental understanding of the forming behaviour and the material flow of an additively built metal part compared to the conventionally manufactured material. Therefore mechanical properties of both materials will be identified by upsetting tests. Additionally, fibre flow and Vickers hardness before and after the forming operation are analysed. It is detected that the forming behaviour of the additively manufactured material is strongly influenced by the layer-wise building process.

Keywords: Additive Manufacturing, Cold Forming, Steel
\end{abstract}

\section{Introduction}

A current trend in the industry is an increasing variety and the individualization of products on costumers desires. This leads to shorter product life cycles and therefore the necessity of highly flexible processes to face the quick changes of the product development. Within this context, laser-based additive manufacturing processes have become more important in the last few decades and changed from a prototyping technology to a production technology [1]. Additive manufacturing, in general, uses the principle that every three dimensional part can be divided up into infinitesimal small layers. As each layer describes the cross section of the part at this specific plane, the part also can be produced by joining these layers one after another [2]. However, the assumption of infinitesimal layers is idealised for real manufacturing processes. The layer thickness is influenced by process parameters like e.g. the size of the powder or the recoating process.

Not just design parts made by plastics can be produced by additive manufacturing but also functional metal parts for example by selective laser beam melting (LBM). Within this process, a thin layer of metal powder is applied onto a building plate by using a recoater. Subsequently, a laser beam melts the powder to manufacture the cross section before the building plate is lowered and the next layer of powder is applied. To prevent the molten metal from oxidation during the process the building plate is placed in a building chamber that is flooded with an inert gas like nitrogen or argon [3]. The advantages of the additive manufacturing technology are the high geometrical freedom for designing components and the high material efficiency as only the needed material for the part is used and remaining powder can be saved for the next building process. The main disadvantages are a poor geometrical accuracy due to the layer-wise production and a rough surface caused by powder particles sticking to the part. Furthermore, the mechanical properties highly depend on process parameters, homogeneity of the powder material, machine maturity and instabilities during the process, therefore, the reproducibility of parts built by LBM is still a challenge. For this reason, the mechanical properties have a high deviation between additively manufactured parts compared to components build by a forging process [4]. The main advantage of forging processes is the formation of a fibre flow and therefore relatively high and constant mechanical properties as well as a high geometrical accuracy for each manufactured part which is favourable for dynamically stressed parts.

Current research within the Collaborative Research Centre 814 "Additive Manufacturing" investigates the combination of additive manufacturing and sheet metal forming. Therefore, functional elements are added onto a sheet metal by LMB and this hybrid part is formed by deep drawing [5] or bending [6] afterwards whereas the

* Corresponding author: thomas.papke@,fau.de 
forming operation is primarily applied to the sheet metal. The aim is a fundamental knowledge of interactions combining these two technologies and to design process chains with a high degree of flexibility and the productivity of forming processes.

The objective of the investigation presented in this work is to analyse the forming behaviour of additively manufactured elements within a bulk metal forming operation. A possible application for this approach would be a hybrid semi-finished product that gets the final shape in a combined sheet metal and bulk metal forming process. In order to determine the forming behaviour of additively manufactured elements by LBM, an upsetting test is conducted as a simple bulk metal forming operation. Using this test the forming behaviour as well as the mechanical properties can be analysed. The material stainless steel 316L (1.4404) is chosen. This steel is common in additive manufacturing and can also be used for cold forging operations. To evaluate the forming of additively manufactured elements they are compared with conventionally manufactured specimens. Additionally, the microstructure and the resulting work hardening after forming are analysed.

\section{Experimental Setup}

\subsection{Laser Beam Melting}

At this point, the manufacturing of high-quality parts with $316 \mathrm{~L}$ is possible with commercially available machine parameter sets for this kind of stainless steel. However, the properties of metal parts manufactured with laser beam melting depend strongly on the powder material used [7]. Hence, the material quality is an important objective in the research of additive manufacturing. The particle size distribution, the chemical composition and the humidity of the powder affect its usage. Therefore an analysis of the used powder is conducted. The humidity is measured with a Karl-Fischer Coulometer (KF 860 Thermoprep) and determined the mass of the contained water is $145.8 \mu \mathrm{g}$ inside a $7.19 \mathrm{~g}$ sample, so the content is below $0.002 \%$. Fig. 1 shows a scanning electron microscope (SEM) image of the used powder.

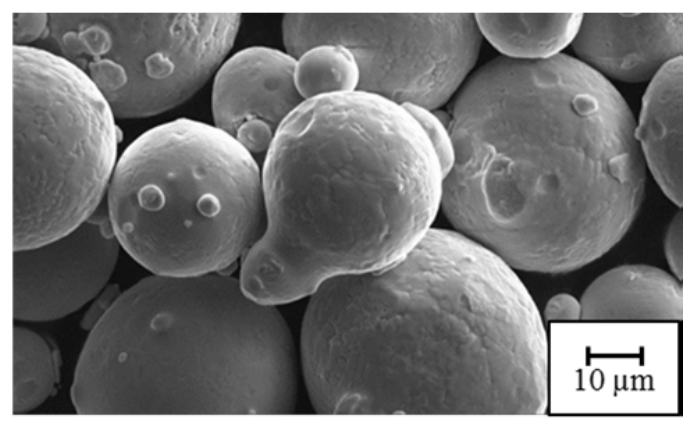

Fig. 1. SEM image of particle shape

The chemical composition is determined with an inductively coupled plasma-optical emission spectroscopy (ICP-OES) and by a combustion analyser at an external testing laboratory. The analysed chemical composition is shown in Table 1. Furthermore, the particle size distribution of $25 \mu \mathrm{m}$ to $45 \mu \mathrm{m}$ is measured by dynamic image analysis (Camsizer Retsch Technologies).

Table 1. Chemical composition analysis of the used powder by plasma-optical emission spectroscopy in wt-\%

\begin{tabular}{|c|c|c|c|c|c|c|c|c|}
\hline $\mathrm{C}$ & $\mathrm{Cr}$ & $\mathrm{Fe}$ & $\mathrm{Mn}$ & Mo & $\mathrm{Ni}$ & $P$ & $\mathrm{~S}$ & $\mathrm{Si}$ \\
\hline$\stackrel{5}{0}$ & $\underset{\infty}{+\infty}$ & $\begin{array}{l}\vec{y} \\
\mathscr{Q} \\
\mathscr{q}\end{array}$ & $\begin{array}{l}\stackrel{R}{a} \\
0\end{array}$ & $\frac{b}{i}$ & 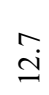 & $\begin{array}{l}\text { Iิ } \\
\text { } \\
\text { Vे }\end{array}$ & 용 & $\begin{array}{l}\text { ర్రి } \\
\text { : }\end{array}$ \\
\hline
\end{tabular}

The test specimens for the bulk forming operation have been produced with a M2 machine of the company Concept Laser $\mathrm{GmbH}$. The machine is equipped with a 400 Watt laser and the models are sliced in $25 \mu \mathrm{m}$ layers. The scan parameters use a hull-core-island strategy according to [8]. To guarantee an isotropic microstructure the scanning orientation is switched for each layer. For a simple comparison, the volumetric energy input $\mathrm{E}_{\mathrm{v}}$ for the different regions is calculated by dividing laser power $\mathrm{P}$ by the multiplication of scan velocity $v$, hatch distance $h$ and the layer height 1 [3] as shown in equation (1):

$$
E_{v}=P /(v h l)
$$

The parameter set of the hull is defined with $E_{\mathrm{v}}=89.4 \mathrm{~J} / \mathrm{mm}^{3}$ and for the core area it is defined with a value of $\mathrm{E}_{\mathrm{v}}=86.6 \mathrm{~J} / \mathrm{mm}^{3}$. The hull-core strategy is often applied by various machine manufacturers since it decreases the manufacturing time as the core can be molten with less accuracy only every second layer. The small layer height of $25 \mu \mathrm{m}$ is chosen in order to produce parts with a relative density higher than $99.9 \%$. To get a constant quality of the additively manufactured material cylinders with a diameter of $50 \mathrm{~mm}$ and a height of $55 \mathrm{~mm}$ are produced. The test specimens are extracted from these cylinders.

To analyse the strength and the forming behaviour of the additively and the conventionally manufactured material $316 \mathrm{~L}$, cylinders with a diameter of $10 \mathrm{~mm}$ and a height of $15 \mathrm{~mm}$ are tested. First, bars with a diameter of $11 \mathrm{~mm}$ are extracted from the additively manufactured cylinder in the longitudinal direction by electrical discharge machining. Second, to reduce surface effects of the electrical discharge machining the final geometry is realized by turning. For the specimen of conventional material, hexagonal bars are taken as raw material and the samples are also manufactured by electrical discharge machining and finished by turning.

\subsection{Forming Test}

To investigate the forming behaviour of additively manufactured material an unidirectional forming is realised by an upsetting test. It allows the identification of mechanical properties and the analysis of the material flow under compression load. 
The upsetting tests, schematically sketched in Fig. 2, are conducted on a universal testing machine LFEM 300 from the company Walter+Bai with a maximum force of $300 \mathrm{kN}$. Due to the high load during compression testing, a carbide tool is used. For constant low friction conditions, a Teflon film is put in between the specimen and the punches. The tests are conducted in accordance with DIN 50106 [9] for upsetting tests with a compression velocity of $5 \mathrm{~mm} / \mathrm{min}$ and the specimen is compressed to $50 \%$ of the initial height or until the set force limit of $200 \mathrm{kN}$ is reached.

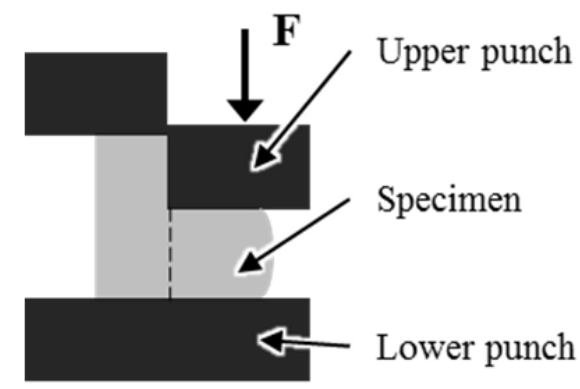

Fig. 2. Schematic principle of upsetting test

To analyse the forming behaviour the stress-strain curve can be determined from the machine data. For avoiding deviations by deflection of the machine frame an elastic correction curve of the machine is recorded prior to the tests. Additionally, the tests are recorded by CCD-Cameras of an ARAMIS System for an in-situ analysis of the forming behaviour.

\subsection{Metallographic Analysis}

In order to investigate the microstructure of additively manufactured and conventionally manufactured material cross sections are analysed after the upsetting tests. After polishing the specimens are etched with V2A-etchant for $20 \mathrm{~s}$ to visualize the microstructure.

\subsection{Hardness Measurement}

For a comprehensive investigation of mechanical properties, the hardness of the specimens is measured before and after the forming process. The measurements are conducted with the Fischerscope HM2000 from the Helmut-Fischer $\mathrm{GmbH}$ that allows a high-resolution measurement of the Vickers Hardness (HV0.5). To investigate the influence of work hardening on the hardness, a grid of measurement points is used. The distance between the grid points is $0.3 \mathrm{~mm}$ in vertical and horizontal direction. The hardness before the forming operation is measured at several points and identified as the initial hardness.

\section{Results and Discussion}

\subsection{Upsetting Test}

To analyse the mechanical properties stress-strain curves are calculated from machine data recorded during the upsetting tests. Fig. 3 shows the curves, representing mean values, of conventionally and additively manufactured material exemplarily.

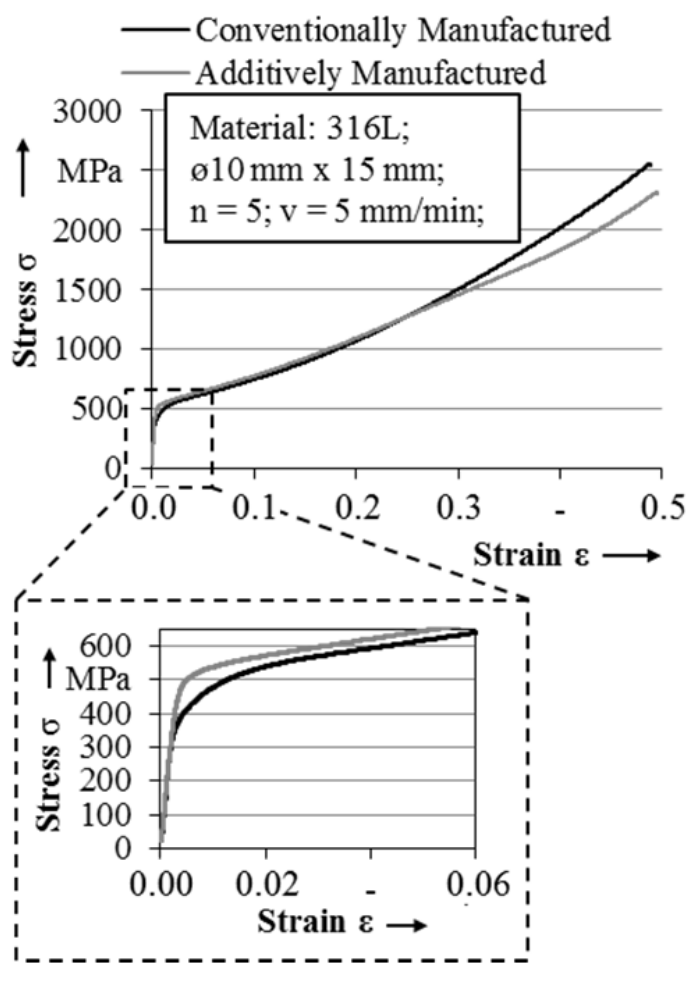

Fig. 3. Stress-strain-curves for conventionally and additively manufactured material calculated from machine data

In the beginning, the elastic behaviour of both materials is similar. In the further evolution of the curve a difference of the yield strength, which represents the beginning of plastic forming, is analysed. The additively manufactured specimen has a yield strength $(508.7 \pm 2.4 \mathrm{MPa})$ which is $25 \%$ higher than that of the conventional material of $408.8 \pm 1.1 \mathrm{MPa}$. Afterwards, the conventional material has a higher work hardening until a strain of 0.02 before the curves are progressing parallel, although the stress of the additively manufactured material is slightly higher (Fig. 3). At a stress value of about $1000 \mathrm{MPa}$ and a strain of about 0.2 the work hardening of the additively manufactured specimen is decreasing and at about $1300 \mathrm{MPa}$ the stress-strain curve crosses that of the conventional material at a strain of about 0.25 . Until the crossing of the curves the materials' properties are reproducible. One reason for the decreasing stress could be shearing effects inside the additively manufactured specimen that ends in the presented distortion after the upsetting test.

To support the explanation of the flow behaviour Fig. 4 gives an overview of the change in shape of the specimens for strains from 0.1 to 0.4 and after the forming test. For strain 0.1 the shape of the specimen for both materials can be described as cylindrical. However, for strain 0.2 a beginning distortion of the specimens' outer shape orthogonal to the forming direction can be determined for the additively manufactured material. In contrast, the shape of the conventionally manufactured material remains cylindrical. This could explain the deviation of the flow curves, which begins at this strain. 
a) Stages of Forming:

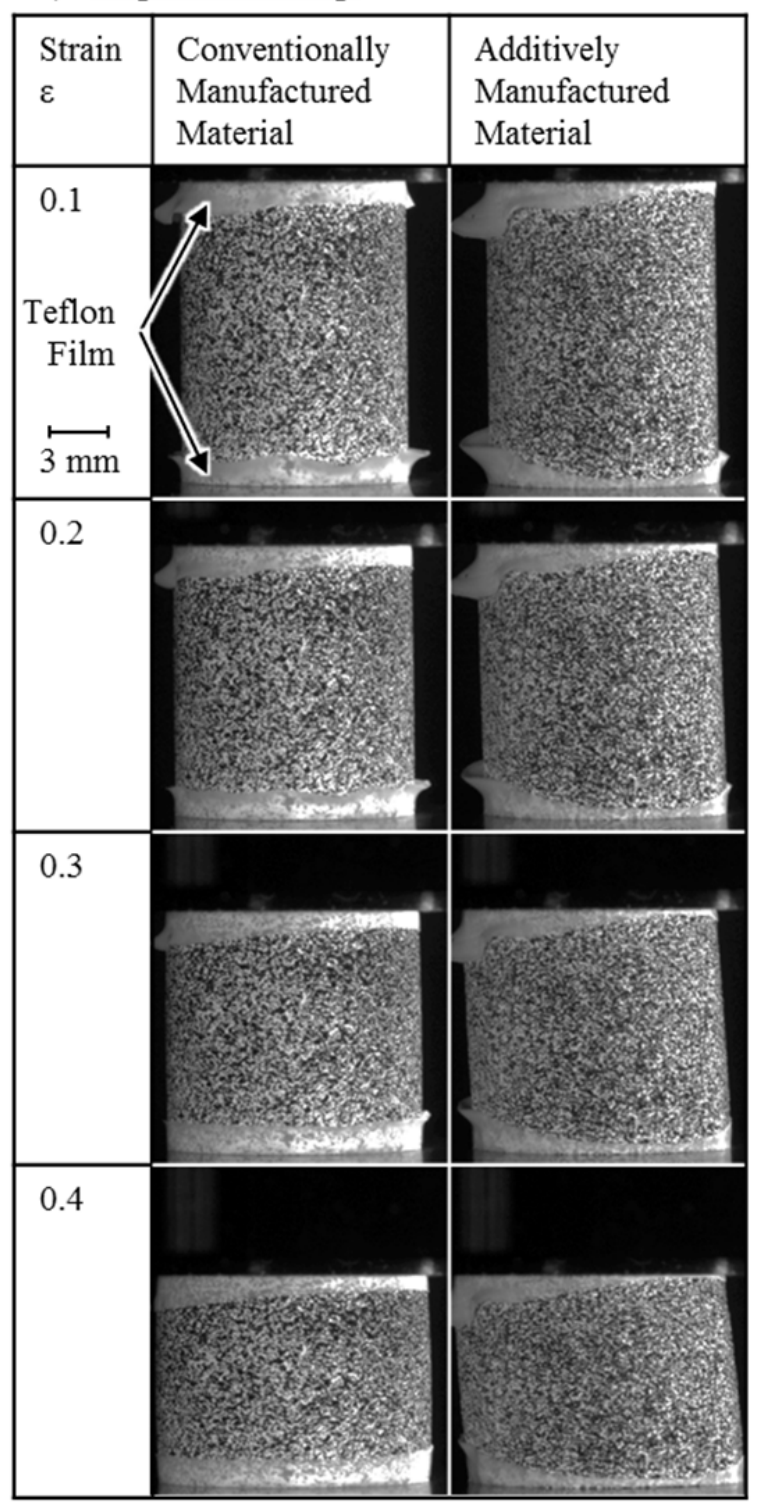

b) After the Upsetting Test

Conventionally Manufactured Material: $\varepsilon=0.5$

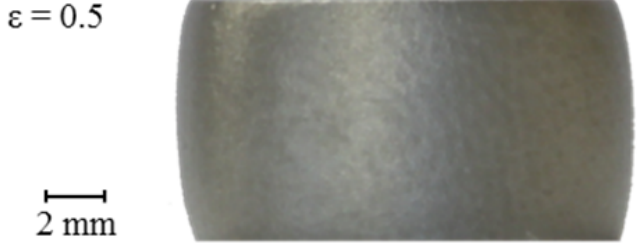

Additively Manufactured Material:

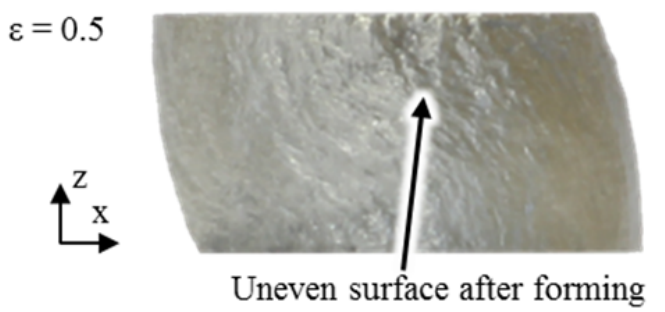

Material: $316 \mathrm{~L}$; Upsetting Test: $5 \mathrm{~mm} / \mathrm{min}$

Fig. 4. Shape of specimens for conventionally and additively manufactured material for a) subsequent stages of forming and b) after the upsetting test
The difference in the shapes increases for higher strain values as 0.3 and 0.4 until the final shape after the forming process is reached. As shown in Fig. 4 b), the specimens of the upsetting tests of the conventionally manufactured material result in a symmetrical cylinder as it is common for this test. Contrary to this, for the upsetting tests of the additively manufactured material a distortion of the specimen is observed for each of the five repetitions, exemplarily shown in Fig. 4 b). Furthermore, an uneven surface of the additively manufactured specimen results from the free forming operation. That indicates a different forming behaviour of the additively manufactured material in contrast to the conventional material.

\subsection{Metallographic Analysis of Flow Behaviour}

It is assumed that the inhomogeneous forming behaviour is caused by the specimen's microstructure, therefore the initial state of the microstructure of conventionally and additively manufactured material is analysed by polished and etched cross sections, shown in Fig. 5.

\section{a) Conventionally Manufactured Material}

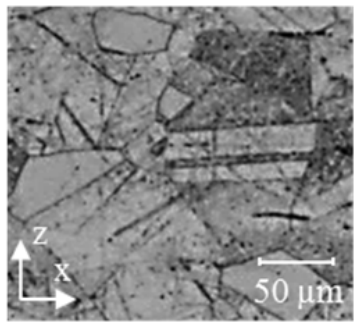

b) Additively Manufactured Material

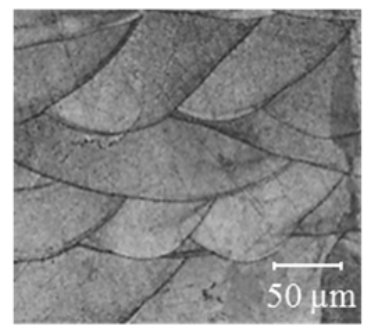

Fig. 5. Analysis of the microstructure before forming of a) conventionally and $\mathrm{b}$ ) additively manufactured material

For the conventional material, a small grained structure can be identified compared to the additively manufactured material. Within the additively manufactured part, the microstructure is dominated by the shape of welding beads representing the single layers, which correspond to the scan track melt pools. In the additively manufactured structure, just a few beads are grown over several adjacent layers.

To analyse the fibre flow due to the upsetting test, the specimens are analysed after the forming process. Fig. 6 shows the fibre flow for the formed conventionally and additively manufactured material.

The fibre flow of the conventionally manufactured material shows the typical shape after an upsetting specimen. Analysing the change of the direction of the fibres relative to the punch direction (z-direction), the microstructure in the cross section can be divided into highly and hardly formed areas. Along the diagonals, slip bands caused by the forming during the upsetting test are identified. However, the fibre flow of the additively manufactured material does not show a similar orientation. Due to the etching of the cross section, the layers caused by the additive manufacturing process are visible. The separation of the microstructure 
in highly and hardly formed areas as it was identified for the conventionally manufactured material is not possible. Based on the orientation of the layers after the upsetting test, a distortion of the upper side of the specimen relative to the lower side along the $\mathrm{x}$-axis can be seen.

\section{a) Conventionally Manufactured Material:}

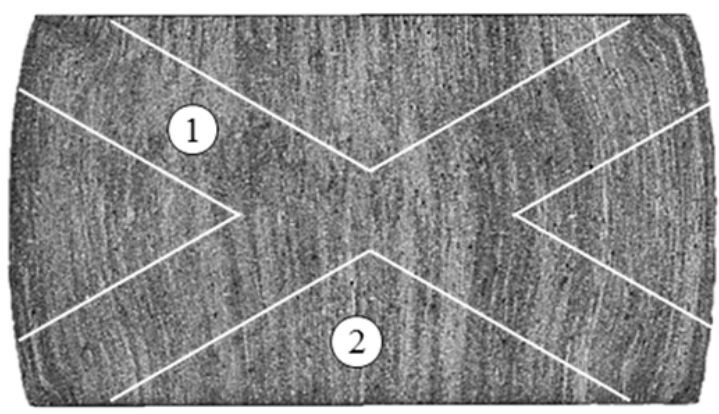

(1) Highly Formed Area (2) Hardly Formed Area

b) Additively Manufactured Material:

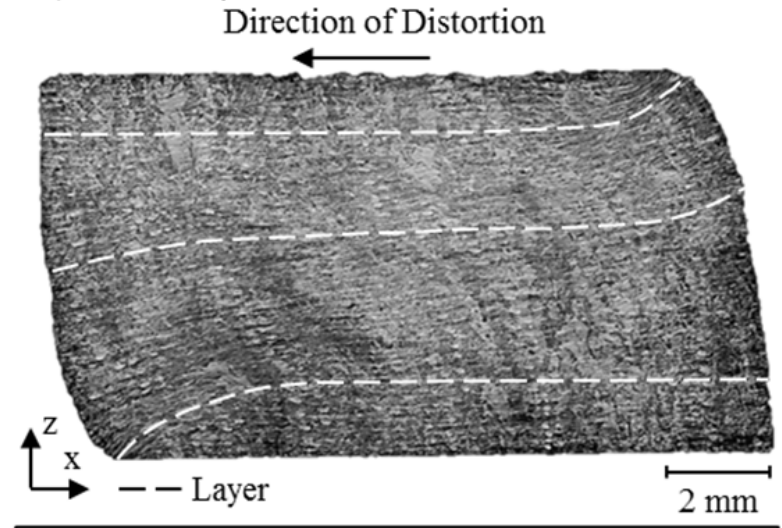

Material: 316L; Etching: V2A-Etchant, $t=20 \mathrm{~s}$

Fig. 6. a) Conventionally and b) additively manufactured etched cross sections of specimens after upsetting tests

An explanation that can be assumed regarding the cross section shown in Fig. 6 b) could be the slide of the layers onto each other due to the forming process. Commonly known for forming is the formation of slip bands under high loads, on which the material slides during deformation [10]. The initiation of slip bands could start at the corners of the specimen in Fig. 6 a), which is indicated due to the diagonal alignment of the fibres. As LBM in principle is a welding process, a heat affected zone results for each layer and causing brittle phases [11]. Thus a possible explanation may be that while a force causes the forming of the specimen the weak areas of the layers begin to deform whereas the brittle phases within the heat affected zones have higher yield strength and resist the deformation. Reaching the maximum formability, the weak areas start to slip onto the brittle layers.

\subsection{Hardness Measurement}

For a comprehensive investigation of the mechanical properties, the Vickers hardness (HV0.5) is measured before and after the forming process. For the initial state, the mean hardness value of the conventionally manufactured material is $245.45 \pm 16.35 \mathrm{HV} 0.5$ compared to $257.30 \pm 15.49 \mathrm{HV} 0.5$ of the additively manufactured material. Therefore, no significant difference can be measured that could explain the difference of $100 \mathrm{MPa}$ between the yield strengths.

A hardness mapping for both materials after the upsetting test is shown in Fig. 7. The measuring grid for specimens of both materials after forming is set to $0.3 \mathrm{~mm}$.

a) Conventionally Manufactured Material:

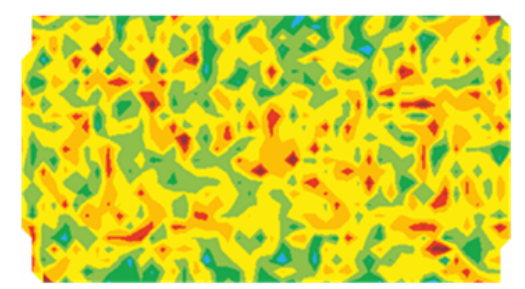

b) Additively Manufactured Material:

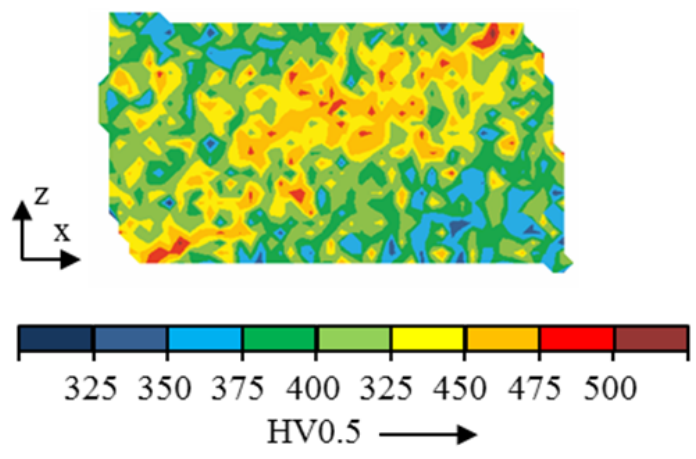

Fig. 7. Mapping of hardness values for a) conventionally manufactured material and $b$ ) additively manufactured material specimens after upsetting test

The analysis of the conventionally manufactured specimen depicted in Fig. 7 a), shows work hardening with a minimum value of $350 \mathrm{HV} 0.5$ within the whole cross section, which is about $100 \mathrm{HV} 0.5$ higher than that measured for the unformed sample. Higher values up to $560 \mathrm{HV} 0.5$ are mainly measured within the highly formed areas. In comparison, the work hardening of the additively manufactured specimen up to more than 500 HV0.5 takes place along the diagonal slip band, whereas the hardness in the adjacent material only increases about $50 \mathrm{HV} 0.5$ to a value of about $300 \mathrm{HV} 0.5$. However, for the additively manufactured material high hardness values are located along the diagonal direction, which is also indicated by the distortion of the layers along the $\mathrm{x}$-axis in Fig. $6 \mathrm{~b}$ ). The increase in hardness could explain the distortion of the upper side of the specimen relative to the lower side.

\section{Conclusion}

Regarding the results of the presented investigations, it can be concluded that the layer-wise structure of 
additively manufactured material influences the forming behaviour. It is obvious that forming of additively manufactured material differs significantly from forming conventionally manufactured material what could be verified by stress-strain curves and the analysis of the microstructure after forming. Although no significant differences in the hardness of the initial materials can be measured, the layer-wise structure caused by the additive manufacturing process has a huge effect on the material flow during forming.

Concluding the presented results, forming of additively manufactured material can be used like conventional material until the maximum strain is lower than 0.2. For higher strains, the forming behaviour becomes significantly different. An analysis of the specimen geometry during the upsetting test shows distortion of the outer shape orthogonal to the forming direction. In contrast, the shape of conventionally manufactured specimen remains symmetrical cylindric due to the forming operation, which is typical for the upsetting test. The change in the forming behaviour of the additively manufactured material could be indicated in the stress-strain-curve as well. For stresses higher than $1300 \mathrm{MPa}$ the work hardening decreases. Therefore, it could be concluded, that for higher deformation the layer-wise structure of the additively manufactured material has to be taken into account. It seems that every layer has to be considered separately and that the heat affected zone appears as a frontier between the layers. To prove this hypothesis, further investigations are necessary for example by analysing the microstructure by a selective electron microscope and electron backscatter diffraction.

\section{Summary and Outlook}

As the individualization of products is a current trend in industry additive manufacturing is getting more interesting for production. One disadvantage of this technology is a poor reproducibility of the mechanical properties compared to those realized by forming processes. Therefore, bulk metal forming of additively manufactured material is investigated within this work.

To analyse the forming behaviour upsetting tests for conventionally manufactured material and additively manufactured material have been carried out. During these tests, a distortion of the additively manufactured material was observed. Furthermore, the stress-strain curves and the material properties such as the Vickers hardness depending on the material flow are determined also. The results show higher yield strength for additively manufactured material compared to the conventionally manufactured material. Afterwards, the flow behaviour is analysed by visualization of the fibre flow after the forming process by investigating etched cross sections of the specimens. It was claimed that the layers of the additively manufactured material show a distortion orthogonal to the forming direction, which could be caused by brittle phases between the layers.

For a comprehensive investigation of the mechanical properties and visualization of the forming zone, a mapping of the Vickers hardness is conducted for both materials after the upsetting test. As expected, the conventionally manufactured material shows higher hardness values in highly formed zones, which are confirmed by the fibre flow. However, the additively manufactured material shows high hardness values in the diagonal direction of the cross section. Therefore, it can be seen that the hardness distribution for both materials after the forming process is different. This result could support the hypothesis that the forming behaviour is influenced by the layer-wise structure.

Further investigation will deal with the analysis of phases and the mechanical properties of the single layers caused by laser additive manufacturing. For fundamental investigation of forming additively manufactured material, the layer-wise structure has to be taken into account. Furthermore, the influence of a forming process on the orientation of the layers should be investigated by bulk metal forming processes.

This research work has been founded by the German Research Foundation (DFG) within the scope of the Collaborative Research Centre 814 “Additive Manufacturing” subproject B5.

\section{References}

1. M.Schmidt, M. Merklein, D. Bourell, D. Dimitrov, T. Hausotte, K. Wegener, L. Overmeyer, F. Vollertsen, G.N. Levy, CIRP Annals 66/2 561-583 (2017)

2. A. Gebhardt, Generative Fertigungsverfahren 4 (2013)

3. W. Meiners, Direktes selektives Laser Sintern einkomponentiger metallischer Werkstoffe (1999)

4. B. Ahuja, A. Schaub, D. Junker, M. Karg, F. Tenner, R. Plettke, M. Merklein, M. Schmidt, Proc. 15th Annual Conference of RAPDASA, 30-42 (2014)

5. A. Schaub, J. Degner, A. Bhrigu, M. Schmidt, M. Merklein, Int. Conf. on Comp. Manuf., (2016)

6. M. Merklein, P. Dubjella, A. Schaub, L. Butzhammer, M. Schmidt 6th Int. Conf. on Add. Tech. 309-316 (2016)

7. A. Cooke, J. Slotwinski, NISTIR 7873 (2012)

8. S. Kumar, J.-P. Kruth, Advanced Engineering Material 10.8 750-753 (2008)

9. DIN50106:2016-11 Testing of metallic materials Compression test at room temperature (2016)

10. K. Lange, Umformtechnik (1984)

11. G. Schulze, Die Metallurgie des Schweißens (2010) 